\title{
Apartment Structure Estimation Using Fully Convolutional Networks and Graph Model
}

\author{
Toshihiko Yamasaki \\ The University of Tokyo \\ yamasaki@hal.t.u-tokyo.ac.jp
}

\author{
Jin Zhang \\ The Univ. of Tokyo and EPFL \\ in.zhang@epfl.ch
}

\author{
Yuki Takada \\ The University of Tokyo \\ takada@hal.t.u-tokyo.ac.jp
}

\begin{abstract}
Apartment searching has been a hot demand all over the world. Understanding the apartment structure will significantly contribute to simplifying the searching process. In this project, the fully convolutional networks are applied to generate semantic segmentation for the apartment floor plan images. We then study and optimize an algorithm to extract the graph model from the segmentation, and to extract the maximum common subgraph with which the structure similarity can be measured. We generate the ground truth data by using online annotation tool and experiment on half of the data. Our segmentation prediction results achieve a pixel mean accuracy of 0.89 for the full-label model, and retrieve quite similar apartment structures by using graph modeling.
\end{abstract}

\section{CCS CONCEPTS}

- Computing methodologies $\rightarrow$ Image segmentation;

\section{KEYWORDS}

Apartment structure, fully convolutional networks (FCNs), graph measure, maximum common subgraph (MCS)

\section{ACM Reference Format:}

Toshihiko Yamasaki, Jin Zhang, and Yuki Takada. 2018. Apartment Structure Estimation Using Fully Convolutional Networks and Graph Model. In Multimedia for RETech'18: Workshop on Multimedia for Real Estate Tech, June 11, 2018, Yokohama, Japan. ACM, New York, NY, USA, 6 pages. https://doi.org/10.1145/ 3210499.3210528

\section{INTRODUCTION}

There has been an increasing demand for apartment searching in recent years, especially in big cities. It is a difficult task because of the tremendous resources. The emergence of online searching platforms has eased this task. However, most of the searching platforms only include information about apartment location, monthly rent, room size, and so on, but little information on apartment structure. This shortcoming takes huge energy if one wants to find an ideal apartment. Thus understanding the apartment structure from the floor

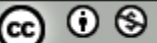

This work is licensed under a Creative Commons Attribution-NonCommercial International 4.0 License.

Multimedia for RETech'18, June 11, 2018, Yokohama, Japan (C) 2018 Copyright held by the owner/author(s).

ACM ISBN 978-1-4503-5797-5/18/06.

https://doi.org/10.1145/3210499.3210528 plan image database and make it user-understandable will do significant help to simplify the searching process.

Related work has been conducted on floor plan image analysis. $[8,14,18]$ analyzed the floor plans for 3D model generation. [6] focused on generation of corresponding CAD format. [15] worked on the architectural floor plan image, and proposed an algorithm to detect rooms from the image. The line detection algorithm is based on the Hough transform with image vectorization. The lines extracted from this algorithm are regarded as wall contours, and are used as the candidates of the wall detection. Then logical analysis is applied on the result of the wall hypothesis, and the room object can be located. The work in [4] is more in detail, and uses much more information from the floor plan image, and the work is improved in [5]. First, various types of information are separated from the floor plan image. In addition to the wall detection and semantic analysis to detect rooms, the room labels are also predicted by analyzing the extracted text or graphic segmentation. However, this method requires a fully labeled dataset. [7] used a statistical method to make pixel-based segmentation, and wall and room entity are extracted by a deep first search algorithm. Lastly rooms are extracted by structural analysis on the entities. All of the works introduced above are focused on line or wall detection, on which semantic or structural analysis is applied to extract the room entity. Room labels are separately predicted by text information extracted from the floor plan image, based on the fact that the text labels extracted from the floor plan images are precise and uniform.

The powerful deep learning tool hasn't been applied to the floor plan analysis area. In addition, these works stop at the point of obtaining the segmented and labeled room entity from the floor plan image, further analysis on the global apartment structure hasn't been conducted. In our work, we first treat the floor plan image analysis as a semantic segmentation problem, which is discussed in $[9,10,17]$ using convolutional nets. The fully convolutional networks (FCNs) proposed in [13] are the first work to train FCNs end-to-end for pixel-wise prediction and from supervised pre-training. We aim to use this efficient deep learning network for the segmentation and labeling of the apartment floor plan images, followed by analysis on graph model representation with similarity measure.

In this paper, we study the FCNs [13] for semantic segmentation to segment each part of the floor plan images with the class it belongs to. To enhance our understanding on the apartment structure from the segmentation, the graph model is extracted from the floor plan segmentation, with 
engineering work to make the graph model clean. To extract the maximum common subgraph (MCS), we find that McGregor's algorithm [16] shows good performance on graphs with the numbers of nodes and edges both around 10, which is just our case. However, McGregor's algorithm can only extract the common graphs, but miss the common vertices that have no neighbors. To simplify the extraction algorithm and to combine it with our similarity measure, we design and optimize a new algorithm to extract the MCS as well as common isolate vertices, and define the similarity computation, which provides the possibility for similar structure retrieval using a query floor plan image.

The contribution of this paper is two folds. First, we propose to apply the empirical FCNs for the floor plan image segmentation. Second, we demonstrate the apartment structure retrieval by introducing the graph model, as well as a newly designed algorithm for MCS extraction. Our experimental results showed that the FCNs work very well on the room location of the floor plans, and that our graph algorithm is able to retrieve floor plans with structures that are quite similar to the query image.

\section{FLOOR PLAN SEGMENTATION}

To solve the semantic segmentation problem as well as pixelwise prediction task, we apply the FCNs [13] for our segmentation work.

\subsection{Fully convolutional networks (FCNs)}

The convolutional neural networks (CNNs) have been proved to be powerful in image classification in [12, 20, 21]. A CNN is usually composed of repeating or nesting convolution units with basic components of convolution, pooling and activation functions. The typical deep nets append fully connected layers to the convolutional networks and compute a linear function of every output of the convolutional layers.

The fully connected layers produce non-spatial outputs for spatial inputs. In the FCNs proposed in [13], the fully connected layers are also regarded as CNNs with a kernel size equal to the entire input region. This enables convolution on any input size and generate classification feature maps.

This initial FCN reduces the input image size by subsampling with kernel stride, making the outputs quite coarse compared to the inputs. So the upsampling structure is proposed to generate dense predictions. Upsampling with a factor $f$ can be regarded as convolution with stride $1 / f$, i.e. backwards convolution (also called deconvolution) with stride $f$.

\subsection{Fine-tune with finer stride}

In the FCNs we apply, the stride for convolution nets is 32. Thus to keep the input and output dimensions equal, the upsampling stride is also set to 32. The coarse FCN32s network borrows the convolution nets from VGG net [20]. The quite downsampled output from the VGG net is then upsampled back to the original size of the input image by a learnable upsampling layer with a stride 32 .
In convolutional networks, the lower convolutional layers are able to capture finer grain structures in the input image because they have a smaller receptive field. So the finer FCNs take different convolutional feature maps in different layers, apply separate learned upsampling to each of them, and combine them together to produce the final output.

\subsection{Measurements}

We evaluate the segmentation results by pixel-level accuracy and object-level accuracy, which are common measurements of semantic segmentation problem.

For pixel-level accuracy, the pixel accuracy and mean accuracy is defined in (1) and (2), respectively. The pixel accuracy is computed over one segmented image.

$$
\begin{aligned}
\text { pixel accuracy } & =\frac{\sum_{i} p_{i}}{\sum_{i} n p_{i}}, \\
\text { pixel mean accuracy } & =\frac{1}{n_{c l}} \sum_{i} \frac{p_{i}}{n p_{i}},
\end{aligned}
$$

where $p_{i}$ is the number of pixels of class $i$ that are correctly predicted, $n p_{i}$ is the total number of pixels of class $i$, and $n_{c l}$ is the total number of classes.

For object-level accuracy, an object is defined to be correctly predicted when over 80 percent of the pixels of the object is correctly predicted. Then the object accuracy and mean accuracy is defined in (3) and (4), respectively. The object accuracy is computed over the whole test dataset.

$$
\begin{aligned}
\text { object accuracy } & =\frac{\sum_{i} o_{i}}{\sum_{i} n o_{i}}, \\
\text { object mean accuracy } & =\frac{1}{n_{c l}} \sum_{i} \frac{o_{i}}{n o_{i}},
\end{aligned}
$$

where $o_{i}$ is the number of objects of class $i$ that are correctly predicted, and $n o_{i}$ is the total number of objects of class $i$.

\section{GRAPH MODEL ON APARTMENT STRUCTURES}

To enhance our understanding on the apartment structure from the segmentation prediction of the FCN, we introduce graph to model the floor plan structure, as well as to measure the structure similarity. In this section, we design and optimize an algorithm to extract the graph model from the floor plan segmentation and to extract the MCS of two structures, and define the similarity measure based on the MCS.

\subsection{MCS extraction}

In the graph model, the independent rooms in the floor plans are represented by the vertices of the graph, and the adjacency of two rooms is represented by the graph edges. The most straightforward way to measure the similarity between any two structures is to measure how much common structure their corresponding graph models share with each other, which leads to the MCS problem. Here we explain the MCS extraction algorithm. 
- Step 1 - adjacency matrix from graph For two graphs $G$ and $H$, any duplicate vertex name in $G$ does not exist in $H$, and vice-versa. The adjacency matrix is the square matrix representing the finite graph, the elements of the graph indicating whether pairs of vertices are adjacent or not in the graph.

\section{- Step 2 - adjacency matrix intersection}

To measure the MCS, an intersection is taken over the vertices of the two graphs by vertex names, according to which the adjacency matrices are clipped into the same dimensionality and arranged in the same vertex sequence as in the intersection.

- Step 3 - matrix multiplication

The multiplication is taken over the two clipped adjacency matrices, and the elements in the multiplication matrix indicate the adjacency of the vertices in both graph $G$ and $H$. Thus this multiplication matrix is the adjacency matrix of the MCS.

- Step 4 - permutation on duplicate vertex names Our solution above is based on the fact that graph $G$ and $H$ contain no common and duplicate vertex names. If graph $G$ contains duplicate vertex name which also exists in $H$, or vice-versa, to enable all the possibilities of the apartment structure search with the MCS, the algorithm needs to be improved.

Firstly, for any duplicate vertex name in one graph, if this vertex name also exists in the other graph, the vertices with this name in both graph are renamed by adding a number indicator. Then for the graph with a larger number of vertices in this name, a permutation of the new names is generated over these vertices, from which new adjacency matrices are obtained on the permutation. Finally, the MCSs are extracted from each pair of the new adjacency matrices of graph $G$ and $H$. Since several MCSs are extracted from graph $G$ and $H$, further analysis can be applied to identify the best MCS.

\subsection{Similarity measure}

With the MCSs extracted from the graph model of the segmented floor plan, a further work of similarity measure is applied to finalize our analysis. The similarity defined here is concerned with the number of common edges in the MCS, as well as the size distance of the vertices in the two graphs corresponded to the vertices in the MCS.

In two graphs $G=\left(V_{g}, E_{g}, S_{g}\right)$ and $H=\left(V_{h}, E_{h}, S_{h}\right)$, and the MCS $M=(V, E, S)$ of graph $G$ and $H$, where $V_{g}, V_{h}, V$ are the sets of the vertices in $G, H$, and $M ; E_{g}, E_{h}, E$ are the sets of edges in $G, H$, and $M ; S_{g}, S_{h}, S$ are the sets of sizes related to the vertices in $G, H$, and $M$, respectively.

For a vertex $v$ in $M$ with corresponding vertices $v_{g}$ and $v_{h}$ in graph $G$ and $H$, and the sizes for the two vertices are $s_{g}$ and $s_{h}$, respectively. The size of $v$ in $M$ is then simply represented by the similarity of vertex $v_{g}$ and $v_{h}$, which is defined as the size ratio of the two vertices in (5). For an edge $e$ with vertex $v_{e 1}$ and $v_{e 2}$ in $M$, corresponding vertices $v_{e 1_{-} g}$ and $v_{e 2_{-} g}$ in graph $G$, and $v_{e 1_{-} h}$ and $v_{e 2 \_}$in graph $H$,
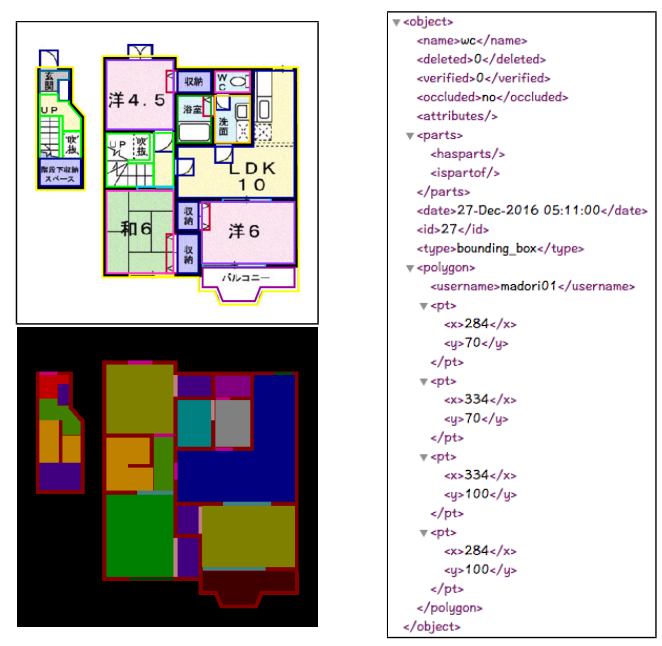

Figure 1: Fully annotated floor plan image with annotation example, and segmentation ground truth extracted from the annotated image

the sizes for the four vertices are $s_{e 1_{-} g}, s_{e 2_{-} g}, s_{e 1_{-} h}, s_{e 2 \_}$. The weight of the edge $e$ is defined as (6).

$$
\begin{gathered}
s(v)=\operatorname{sim}\left(s_{g}, s_{h}\right)=\frac{\min \left(s_{g}, s_{h}\right)}{\max \left(s_{g}, s_{h}\right)}, \\
w(e)=2 \times e^{s\left(v_{e 1}\right)} \times e^{s\left(v_{e 2}\right)} .
\end{gathered}
$$

And then the similarity between graph $G$ and $H$ is defined as the sum of the edge weights and the sizes of the vertices without adjacent vertices in the MCS as in (7).

$$
\operatorname{sim}(G, H)=\sum_{\text {ein } M} w(e)+\sum_{\text {isolatevin } M} s(v) .
$$

\section{EXPERIMENTS AND RESULTS}

\subsection{Dataset}

LIFULL Co., Ltd. released the Home's dataset [1, 3] with the cooperation of National Institute of Informatics, Japan for academic research use. This dataset contains information for more than 5 million apartments, such as monthly rent, area, location, years of age, floor plan, indoor photographs, and so on. We select 5, 000 floor plan images from this apartment information database for our research use. The left-top image in Figure 1 gives an example of the floor plan image dataset.

To generate the ground truth data for our floor plan images, we employ the online annotation tool LabelMe [19] to annotate the floor plan images. We make all the documentation for data annotation and conduct this work through the Crowd Works [2] platform. The ground truth data were annotated by 10 workers. Since the semantic segmentation labeling is very intensive work, it took about two months and cost about US $\$ 5,000$ to generate them.

In our dataset, the floor plans are usually consisted of wall, rooms, washing places, entrance space, storage place, doors, and spaces that are not indicated or accessible. According to 
these different parts, we define 17 labels shown below for our image annotation, among which 12 label classes are used for our full-label model experiments (entrance, rouka, stairs merged as entrance; fan, slide, fold, window merged as door), and six least ambiguous label classes of tatami, west, $d k, w c, b a t h$, washing together with wall and unknown for the six-label model experiments.

\section{Label definition:}

wall: the wall part, usually in black, circling the apartment and separating independent room space;

tatami: the Japanese-style room, usually marked as "tatami", “和室”, “和”, or simply marked in brick shape and possibly with green color;

west: the western-style room, usually marked as "west", “洋室”, or “洋”;

$\boldsymbol{d} \boldsymbol{k}$ : kitchen and/or dining room and/or living room, usually marked as "LDK", "DK", "K", "LK", or firing place figure; $\boldsymbol{w c}$ : toilet, marked as "WC", “toilet", “トイレ”, or toilet figure;

bath: shower room, marked as "bath" or bath pool figure; washing: washing place, marked as “脱衣室” or sink figure; balcony: balcony, marked as "balcony", “バルコニー", or “テラス";

entrance: room entrance, marked as "entrance" or "玄関”; rouka: corridor, marked as “廊下” or “ろうか”;

stairs: up and down stairs, marked as "up", "dn" or stair figure;

cl: closet, shoe case, cloth box, storage room, marked as "cl", “物入”, “下駄箱”, “押入”, “収納”;

fan: fanning door, marked with fan shape;

slide: sliding door, marked with slide door shape;

fold: folding door, marked with fold shape;

window: room window;

unknown: inaccessible or not indicated space, usually blank area or marked with cross.

Figure 1 shows the floor plan image annotation of the example given above with an example of part of the annotation file, and the segmentation ground truth extracted from the annotated image.

In our experiments, 1,635 images are used for training, 500 images for validation, and 500 images for test. This is a relatively small dataset compared to most of the deep learning experiments. However, the average number of labels for each image is about 33 . Therefore, this is a reasonable number for our research. A serious label imbalance also exists in our dataset. We try to penalize on class weights in the experiments, but this gives us no obvious information on the improvement of network performance.

\subsection{Floor plan segmentation}

We run the FCN on this subset of 2, 653 images. The segmentation experiments are conducted under the Caffe framework [11].

Results on six-label model The FCN32s network with a deconvolution layer of stride 32 is quite coarse. The FCN16s network replaces the deconvolution layer of stride 32 with

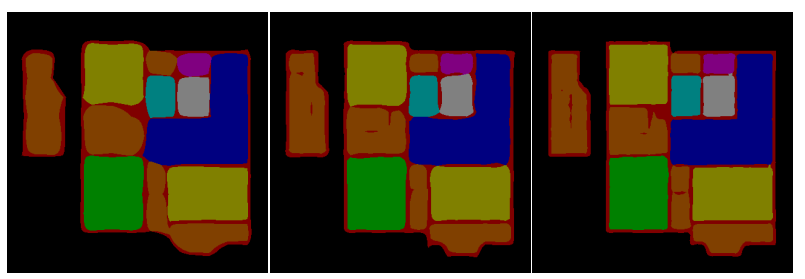

(a) FCN 32 stride(b) FCN 16 stride(c) FCN 8 stride segmentation segmentation segmentation

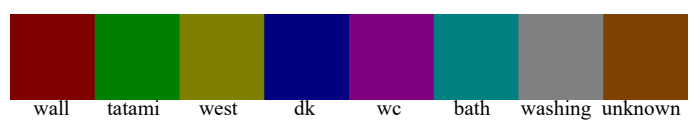

(d) Label color map

Figure 2: A random example from test dataset of 6-label model segmentation prediction

Table 1: Average pixel accuracy for each label class on test dataset for 6-label model, FCN8s

\begin{tabular}{|c|c|c|c|c|}
\hline labels & wall & tatami & west & dk \\
\hline pixel accuracy & 0.8510 & 0.9725 & 0.9869 & 0.9789 \\
\hline \hline labels & wc & bath & washing & unknown \\
\hline pixel accuracy & 0.9532 & 0.9631 & 0.9441 & 0.9285 \\
\hline
\end{tabular}

two deconvolution layers of stride 2 and stride 16, respectively, and the FCN8s replaces the deconvolution layer of stride 32 with 3 deconvolution layers of stride 2, stride 2, and stride 8 , respectively. These fine nets improve the network performance a lot. We also tried to make the network finer, however, we don't find significant improvements by this means.

The pixel accuracy for each class is given in Table 1, and the pixel mean accuracy we obtain is 0.9473 for the six-class model.

Results on full-label model However, the network can fail in some cases, especially in full-label model when the ambiguity of labels is introduced. Figure 3 gives another example. In the original image, blank areas without any feature are indicated as label $c l$ and balcony. In FCN segmentation predictions, they are predicted most likely to be unknown. In Figure 3d, we replace the FCN predictions of the highest probabilities which are not consistent with the segmentation ground truth with second highest probable predictions, and most of the time, the correct labels can take their place back.

One of the shortcomings of the FCN network on the floor plan segmentation is the wall-detection part. As we can see from the above examples, there is still a distance between wall edge predictions and the straight lines in the annotation. When we try to check on the wrong predictions, replacing them with labels of second highest probability, as shown in Figure 3d, the wall edges become much closer to straight lines, which means that the FCN network's performance on the task of separating the rooms and the wall is still to be improved, and this vagueness of lines is caused 


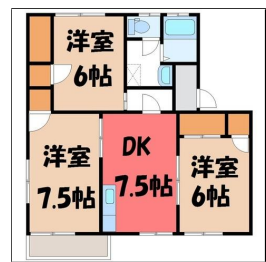

(a) Original floor plan

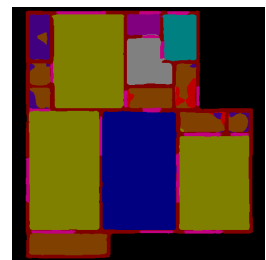

(c) FCN8s

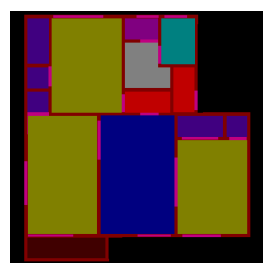

(b) Ground truth

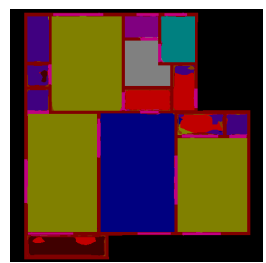

(d) Edited

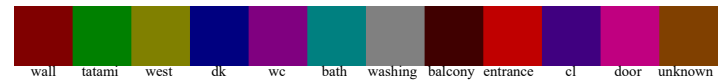

(e) Label color map

Figure 3: Another random example, in 3d the wrong prediction with highest predictions are replaced by the secondhighest prediction

Table 2: Average pixel accuracy for each label class on test dataset for full-label model, FCN8s

\begin{tabular}{|c|c|c|c|c|}
\hline labels & wall & tatami & west & dk \\
\hline pixel accuracy & 0.8199 & 0.9753 & 0.9866 & 0.9782 \\
\hline labels & wc & bath & washing & balcony \\
\hline pixel accuracy & 0.9542 & 0.9657 & 0.9528 & 0.9513 \\
\hline labels & entrance & cl & door & unknown \\
\hline pixel accuracy & 0.8968 & 0.9193 & 0.8114 & 0.5096 \\
\hline
\end{tabular}

by the possibility function within the network that cannot be easily avoided, while its performance on label locating is quite satisfactory.

The pixel accuracy of full-label FCN8s for each class is given in Table 2. We obtain a pixel mean accuracy of 0.8934 on the test data for the full-label model. This is lower than the six-class model because of the ambiguity of the rest labels. And the unknown label accuracy is also significantly decreased. However, all the class accuracy except that of the label unknown is still not bad.

In addition to pixel-level accuracy, we also take a look into the object-level accuracy. The class-wise object-level accuracy is given in Table 3 , and the object mean accuracy is 0.8402 . It is obvious to see that the accuracy on label wall and door is very bad due to the fine and thin characteristic we discuss before. Accuracy for ambiguous label unknown is also bad. While the segmentation on most of the room labels is above 0.85 and even the best ones close to 0.99. Figure 4 gives the comparison of these two kinds of accuracy. Some object-level accuracy is even better than the pixel-level accuracy.
Table 3: Object accuracy for each label class on test dataset for full-label model, FCN8s

\begin{tabular}{|c|c|c|c|c|}
\hline labels & wall & tatami & west & $\mathrm{dk}$ \\
\hline object accuracy & 0.5392 & 0.9836 & 0.9942 & 0.9902 \\
\hline labels & wc & bath & washing & balcony \\
\hline object accuracy & 0.9505 & 0.9659 & 0.9597 & 0.9226 \\
\hline labels & entrance & cl & door & unknown \\
\hline object accuracy & 0.9034 & 0.8586 & 0.6578 & 0.3569 \\
\hline
\end{tabular}

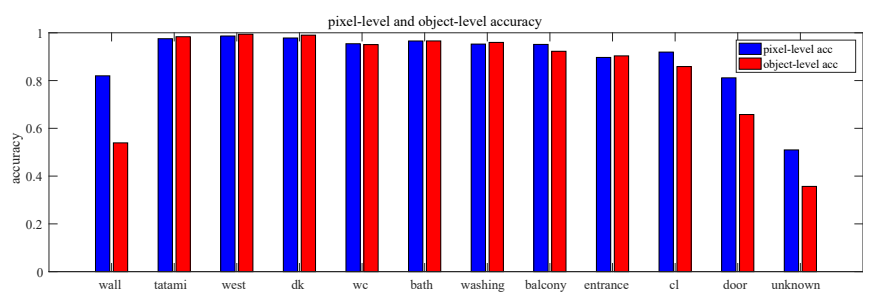

Figure 4: Comparison of pixel-level and object-level accuracy for full-label model

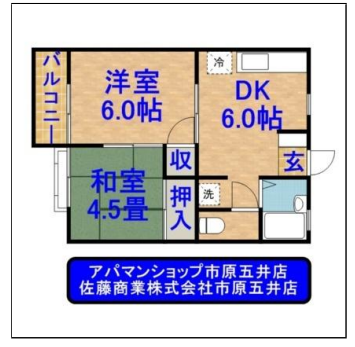

(a) Apartment floor plan image

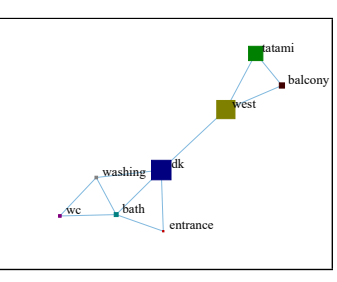

(b) Graph model
Figure 5: Example of graph model extracted from the segmentation ground truth

\subsection{Graph modeling}

Graph models can be extracted form the floor plan segmentation, and floor plans with similar structures can be retrieved by the similarity measure on the MCS.

In the segmented results, to make our graph model clean, an object is considered as vertex if the object size is larger than a threshold, which is set as 1,000. If the minimum distance of two objects is smaller than a threshold, for example, 30 in our experiments, the two vertices the objects correspond to are connected by adding an edge.

For the graph model explanation, we give a simple example here. Figure 5 shows the the apartment floor plan image, with a graph model extracted from it, the vertex sizes indicating the room sizes, and edges indicating adjacent rooms connected.

Figure 6 gives the apartment floor plan images corresponded to the retrieved graph models. Even though the graph model of the segmentation prediction for the query image does not rank the first because of the label prediction failure, it is not far away. And in the retrieved four floor plan 

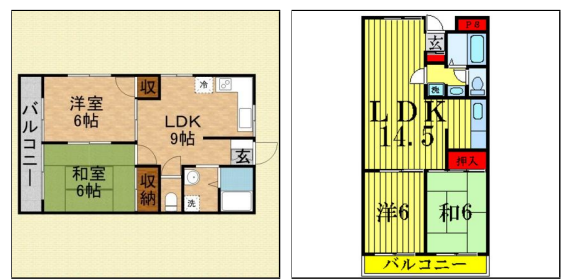

(a) 1st retrieved

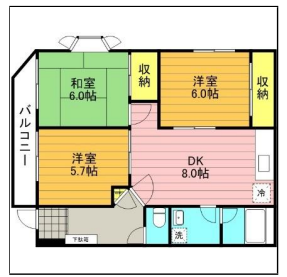

(c) 3rd retrieved (b) 2nd retrieved

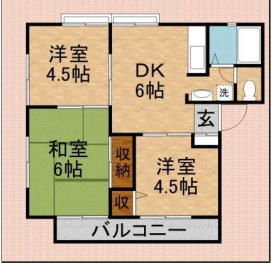

(d) 4th retrieved
Figure 6: Retrieved apartment floor plan image by MCS and similarity measure

images, the apartments enjoy similar structure, with $d k$ in center, west and tatami in one side connected balcony to the end, and bath, wc, washing, entrance to the other side.

\section{CONCLUSION}

In this paper, to achieve the apartment structure estimation, we constructed the annotation ground truth for the floor plan image dataset, which took about two months to finish. This was also the reason that we used half of the dataset for our experiments. Our initial aim was to apply the powerful deep learning tool on the floor plan image, to analyze the apartment structures. When the segmentation results were obtained from the FCN, we further introduced the graph model to make the segmentation more specific and meaningful to usual users, as well as to make our system complete and functional in application use. We designed and optimized the MCS extraction algorithm and defined the similarity measure based on it to make it applicable to the apartment structure searching problem as introduced in the first place. According to the experiment results, our system can measure the apartment structure quite precisely and retrieve apartment floor plan images that are quite similar to the query image.

\section{ACKNOWLEDGMENTS}

In this research, we give thanks to the Next Co., Ltd. for providing the Home's dataset for research use with the corporation of the National Institute of Informatics, Japan.

This work was partially supported by the Grants-in-Aid for Scientific Research (no. 26700008) from JSPS, JST-CREST (JPMJCR1686), and Microsoft IJARC core13.

\section{REFERENCES}

[1] 2010-2017. National Institute of Informatics, Japan. http://www. nii.ac.jp/dsc/idr/next/homes.html. (2010-2017).
[2] 2011-2016. Crowd Works Inc. https://crowdworks.jp/. (20112016).

[3] 2017. NEXT Co., Ltd. http://www.homes.co.jp/. (2017).

[4] Sheraz Ahmed, Marcus Liwicki, Markus Weber, and Andreas Dengel. 2011. Improved automatic analysis of architectural floor plans. In Proceedings of International Conference on Document Analysis and Recognition (ICDAR). IEEE, 864-869.

[5] Sheraz Ahmed, Marcus Liwicki, Markus Weber, and Andreas Dengel. 2012. Automatic room detection and room labeling from architectural floor plans. In Proceedings of 10th IAPR International Workshop on Document Analysis Systems (DAS). IEEE, 339-343.

[6] Yasuhiro Aoki, Akio Shio, Hiroyuki Arai, and Kazumi Odaka. 1996. A prototype system for interpreting hand-sketched floor plans. In Proceedings of the 13th International Conference on Pattern Recognition, Vol. 3. IEEE, 747-751.

[7] Lluís-Pere de las Heras, Sheraz Ahmed, Marcus Liwicki, Ernest Valveny, and Gemma Sánchez. 2014. Statistical segmentation and structural recognition for floor plan interpretation. International Journal on Document Analysis and Recognition (IJDAR) 17, 3 (2014), 221-237.

[8] Philippe Dosch and Gérald Masini. 1999. Reconstruction of the $3 \mathrm{~d}$ structure of a building from the $2 \mathrm{~d}$ drawings of its floors. In Proceedings of the Fifth International Conference on Document Analysis and Recognition. IEEE, 487-490.

[9] Yaroslav Ganin and Victor Lempitsky. 2014. $\mathrm{N}^{\wedge}$ 4-fields: Neural network nearest neighbor fields for image transforms. In Asian Conference on Computer Vision. Springer, 536-551.

[10] Bharath Hariharan, Pablo Arbeláez, Ross Girshick, and Jitendra Malik. 2014. Simultaneous detection and segmentation. In European Conference on Computer Vision. Springer, 297-312.

[11] Yangqing Jia, Evan Shelhamer, Jeff Donahue, Sergey Karayev, Jonathan Long, Ross Girshick, Sergio Guadarrama, and Trevor Darrell. 2014. Caffe: Convolutional Architecture for Fast Feature Embedding. arXiv preprint arXiv:1408.5093 (2014).

[12] Alex Krizhevsky, Ilya Sutskever, and Geoffrey E Hinton. 2012 Imagenet classification with deep convolutional neural networks. In Advances in neural information processing systems. 10971105.

[13] Jonathan Long, Evan Shelhamer, and Trevor Darrell. 2015. Fully convolutional networks for semantic segmentation. In Proceedings of the IEEE Conference on Computer Vision and Pattern Recognition. 3431-3440.

[14] Tong Lu, Huafei Yang, Ruoyu Yang, and Shijie Cai. 2007. Automatic analysis and integration of architectural drawings. International Journal on Document Analysis and Recognition 9, 1 (2007), 31-47.

[15] Sébastien Macé, Hervé Locteau, Ernest Valveny, and Salvatore Tabbone. 2010. A system to detect rooms in architectural floor plan images. In Proceedings of the 9th IAPR International Workshop on Document Analysis Systems. ACM, 167-174.

[16] James J McGregor. 1982. Backtrack search algorithms and the maximal common subgraph problem. Software: Practice and Experience 12, 1 (1982), 23-34.

[17] Feng Ning, Damien Delhomme, Yann LeCun, Fabio Piano, Léon Bottou, and Paolo Emilio Barbano. 2005. Toward automatic phenotyping of developing embryos from videos. IEEE Transactions on Image Processing 14, 9 (2005), 1360-1371.

[18] Siu-hang Or, Kin-Hong Wong, Ying-kin Yu, Michael Ming-yuan Chang, and H Kong. 2005. Highly automatic approach to architectural floorplan image understanding \& model generation. Pattern Recognition (2005), 25-32.

[19] Bryan C Russell, Antonio Torralba, Kevin P Murphy, and William T Freeman. 2008. LabelMe: a database and web-based tool for image annotation. International Journal of Computer Vision 77, 1-3 (2008), 157-173.

[20] Karen Simonyan and Andrew Zisserman. 2014. Very deep convolutional networks for large-scale image recognition. arXiv preprint arXiv:1409.1556 (2014).

[21] Christian Szegedy, Wei Liu, Yangqing Jia, Pierre Sermanet, Scott Reed, Dragomir Anguelov, Dumitru Erhan, Vincent Vanhoucke, and Andrew Rabinovich. 2015. Going deeper with convolutions. In Proceedings of the IEEE Conference on Computer Vision and Pattern Recognition. 1-9. 